\title{
Concentration waves behaviour and displacement development in nanocomposites during mass transfer kinetics on the basis of the bi-functional multicomponent models developed
}

\section{Abstract}

There is realized the theoretical investigation of the multi( $\boldsymbol{n}=\mathbf{6})$-component Mass Transfer (MMT) kinetic process inside the recent combined "NanoComposites (NC)" sorption materials. The NC examples are represented in the manuscript by the bi-functional selective "zero charged $\mathrm{Metal}^{0}\left(\mathrm{NP}^{0}\right)$ Ion Exchangers" NC matrix where the inner active centers, "Nano-Particles ${ }^{0}$ $\left(\mathrm{NP}^{0}\right)$ or in other words " $\mathrm{NP}^{0}$ - nanosites" conditioned as "the agglomerates of the " $\mathrm{NP}^{0}\left(\mathrm{Me}^{0}\right)$ ". The $\mathrm{NP}^{0}$-agglomerates are imbedded into the IEx matrix during the preliminary synthesis of the resulting bi-functional NC matrix.

There is considered the MMT NC kinetics across the bi-functional NC planar L-matrix via the computerized numerical simulation by the mathematical solution of the mass balance, expressed by the partial differential $\boldsymbol{n}(\mathbf{6})$-Eqns. The recent computer numerical simulation approach for the MMT NC kinetics considered here is based on the foundations of the irreversible thermodynamics such as: multi $(\boldsymbol{n})$-component ( $\boldsymbol{n}=\mathbf{6})$ mass balance $\boldsymbol{n}$-Eqns. mentioned; the $\mathbf{k}^{(2)}$ (6)-variance (where $\mathbf{k}^{(2)}$ is determined as "the degree of freedom" for the MMT NC sorption system considered here by the modeling); the fundamental wave concept $(\mathbf{W})$ of the propagating mode of the multi-component $\left\{\mathbf{X}_{n}(\mathrm{~L}, \mathbf{T})\right\}-$ concentration waves; two Mass Action Laws $\left(\mathbf{M A L}_{\mathrm{s}}\right)$ with the $\left\{\mathrm{K}_{\mathrm{s}}{ }^{n}{ }^{n}, 2\right\}-$ two Selectivity parameters; the "sink-source" (or in other words "sorptiondesorption") mass transformation mechanism for various $\mathbf{J}_{\mathbf{k}, \mathbf{m}, \mathbf{p}}$-mass fluxes, and including some others.

The theoretical computer simulation is based on the recent advanced $b i-$ functional $\mathbf{N C}$ Model developed. It comprises the various I, II-MMT co-routes for the two ${ }_{(3,4)} \mathrm{p}$-principal sorbate components in the bi-functional $\mathbf{N C}$ matrix, i.e. (a) the paired $\left(\mathbf{I}_{1,2}\right)$-selective MMT routes described by the $\operatorname{MAL}_{\mathrm{S}}\left(\mathbf{I}_{\mathbf{I}} \mathbf{K}_{1,2}\right)-$ relationships which comprise the two selective paired "mass transformations" \{namely ${ }_{(3.4)} \mathrm{p} \rightleftharpoons \mathbf{m}_{1,2}$ \} onto the active fixed ${ }_{6} \mathbf{R}^{0},{ }^{0} \mathrm{NP}^{0}$-nanosites" (i.e. the zero charged ${ }_{6} \mathbf{R}^{0}$-principal 6th-component, namely "NP0 ${ }^{0}$ nanosites" introduced into the consideration of the multicomponent kinetic process) together with (b) the $\mathbf{D}_{3,4,5}$-multiDiffusion, (II)-MMT co-route for the diffusible ${ }_{(3,4)} \mathrm{p}$-sorbate principals in the NC pores. The $\mathbf{k}^{(2)}(\mathbf{6})$-"variance" of the advanced recent $\mathbf{k}^{(2)}$

Abbreviations: NC, nanocomposites; DD, displacement development; NP, nano-sites

\section{Highlights}

a. Computer simulation by the mass balance Eqns. includes the author's recent nano-composite (NC) Models

b. Numerical solution results bring the propagating mode of the multi-concentration profiles inside $\mathbf{N C}$

c. Phenomenological wave $\mathrm{W}$-concept is extended to a sorption transport phenomena in NC (NanoComposites)

d. Calculated visual animations show the interference of concentration waves for components

e. Simulation results show visually the chromatographic displacement in the $\mathrm{NC}$ via the wave $\mathrm{W}$-concept
Volume 2 Issue 4 - 2018

Kalinitchev Al
Russian Academic of Science, Russia

Correspondence: Kalinitchev Al, Institute of Physics, Chemistry \& Electro Chemistry, Russian Academic of Science, Russia,Email kalin_phyche@mail.ru

Received: March 28, 2018| Published: August 16, 2018

(6)-bi-functional NC Model developed here (including the I\&II-MMT coroutes) is one of the thermodynamic key parameter of the six(n)-component MMT NC sorption system considered.

There is included here into the computer modelling the second MMT (II)-coroute with the realization of the $\left\{\mathbf{D}_{35}\right\}$-multi-Diffusion for the two diffusing ${ }_{(3,4)} \mathrm{p}^{+}$-principal sorbate components including additionally the $\mathbf{5}$ th- anionic co-ions, (i.e. $\mathbf{j}_{5}^{-}$-component intended for the maintaining of the electroneutrality condition) in the combined bi-functional MMT NC sorption system mentioned. The conceptual schemes of the author's advanced $\mathbf{k}^{(2)}(6)-\mathbf{N C}$ Model are displayed in the visual conceptual illustrations with the inclusion of the selective "sink-source" $\mathbf{J}_{\mathbf{k}, \mathbf{m}, \mathrm{p}}$-mass fluxes mechanism mentioned.

Further there is shown visually the interaction of the $\left\{\mathbf{X}_{\mathbf{1 , 2 , 6}}(L, \mathbf{T})\right\}$-travelling concentration waves by the recent computer simulation developed which brings the Displacement Development (DD)-behavior of the two $\mathbf{m}_{1,2}$-interactive $\mathbf{X}_{1}(L, \mathbf{T})$-displacer, and $\mathbf{X}_{2}(\mathrm{~L}, \mathbf{T})$-displaced concentration waves. The intensity of the DD-effect is estimated by the $\mathrm{X}_{2}{ }^{\mathrm{m}}$-peak concentration with depending on the determinative two components' $\left\{\mathrm{K}_{\mathrm{S}}{ }^{\mathrm{m} 1,2}\right\}$-Selectivity factor.

Keywords: concentration waves interaction, selectivity, peculiar waves, bifunctional NC models elaborated, computer simulation, video animation files

\section{Introduction}

The author's computer simulation of the $\operatorname{multi}(\boldsymbol{n}=\mathbf{6})-$ component mass transfer (MMT) NC kinetics is based on the irreversible thermodynamics approach with the mathematical approximate numerical solution of the multi-component mass balance described by the mass balance partial differential MMT $\boldsymbol{n}$-Eqns (with $\mathbf{k}^{(2)}=\mathbf{6}$ variance) for the bi-functional MMT combined NC sorption system previously considered in publications..$^{1-3}$ One of the most important concept of the irreversible thermodynamics used here during the computer simulation proposed is the propagating mode of the multi $(\boldsymbol{n}=\mathbf{6})$-component $\left\{\mathbf{X}_{n}(L, \mathbf{T})\right\}$-concentration waves (S.4,6-8) describing the MMT NC kinetics accordingly to the wave (W)concept. ${ }^{1-3}$

The following notation " $\left\{2_{\mathrm{p}} \mathrm{x}_{\mathrm{m}}\right\}$ below emphasizes the noval expanded $\operatorname{six}(\boldsymbol{n}=\mathbf{6})$-component advanced bi-functional MMT NC Model mentioned with the $\mathbf{k}^{(2)}(\mathbf{6})$-variance comprising the two 
paired $\left\{{ }_{(3,4)} \mathrm{p}^{\mathrm{X}} \mathbf{m}_{1,2}\right\}$ (or else $\left\{2_{\mathrm{p}}^{\mathrm{x}} \mathbf{2}_{\mathrm{m}}\right\}$ ) principal sorption components participated in the MMT selective paired $\left(\mathbf{I}_{1,2}\right)-\mathrm{MAL}_{\mathrm{S}}$ reactions. The $\mathbf{k}^{(2)}(\mathbf{6})-\left\{2_{\mathrm{p}}{ }^{\mathrm{x}} \mathbf{2}_{\mathrm{m}}\right\}$ bi-functional NC Model concerned comprises the corresponding paired selective $\left(\mathbf{I} \cdot \mathrm{K}_{1,2}\right)-\mathrm{MAL}_{\mathrm{S}}$ relationships. The visual conceptual illustrations of the recent $\mathbf{k}^{(2)}(6)-\left\{2_{\mathrm{p}} \mathbf{x}_{\mathrm{m}}\right\}$ NC Model developed are shown below Figure 1.

\begin{tabular}{|c|c|}
\hline \begin{tabular}{l|l}
$(2)$ & bi- functionality : Selectivity \& \\
$\mathrm{K}^{-}$-NC Model & NC MODEL CONCEPT: [] \&
\end{tabular} & $\begin{array}{l}\text { DIFFUSION } \\
\text { III }\left\{\mathbf{D}_{\mathrm{p}, j}\right\}\end{array}$ \\
\hline $\mathrm{m}_{1,2}$ Ia association! dissociation Id & $m_{1,2}$ complexes \\
\hline $\mathbf{J}_{\mathbf{k}}(\mathrm{I} a)<\mathbf{0} \mathrm{m}^{(\mathrm{Rp})^{t}} \mathrm{~m}^{(\mathrm{Rp})^{t}} \quad \mathbf{J}_{\mathbf{k}}(\mathrm{Id})>$ & \\
\hline 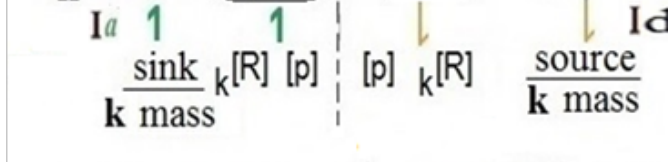 & $\begin{array}{c}\text { two fixed } \\
\text { principal } \\
\text { complexes - } \\
\text { - reagents }\end{array}$ \\
\hline 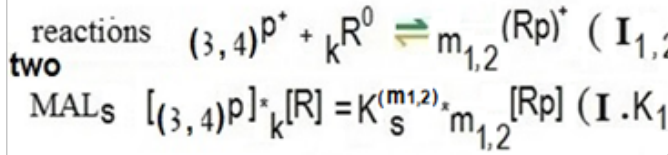 & $\mathrm{m}_{1}$ \\
\hline $\begin{array}{l}\text { Multi component DIFFUSION }\left\{\mathbf{D}_{\mathrm{p}, j}\right\} \text { Mass Transfer } \\
\qquad\left\{\mathrm{D}_{\mathrm{n}}\right\}=\left\{\mathrm{D}_{1,2}=0 ; \mathrm{D}_{3,4,5} ; \mathrm{D}_{\mathrm{k}}=0\right\}\end{array}$ & 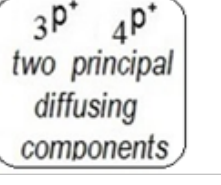 \\
\hline
\end{tabular}

Figure I Recent noval $\mathbf{k}^{(2)}(6)-\left\{2_{\mathrm{p}}{ }^{2} \mathbf{2}_{\mathrm{m}}\right\}$ bi-functional NC Model. Conceptual schemes illustrating (I,II)-MMT co-routes; "sink"-"source" key $\mathbf{J}_{\mathbf{k}^{\prime} \mathrm{m}^{\prime} \text {-mass }}$ fluxes mechanism (upstairs, $\left.\boldsymbol{J}_{\mathbf{k}}>0 \mathrm{r}<0\right)$; $\mathrm{MAI}_{\mathrm{S}}\left(\mathbf{I}_{\mathrm{K}} \mathrm{K}_{1,2}\right)$-relationships; indiffusible ${ }_{k(6)} \mathrm{R}^{0}$-nano-sites, paired $\left\{{ }_{(3,4)} \mathrm{P}^{\times} \mathbf{m}_{1,2}\right\}$-principals; $\left\{\mathrm{K}_{\mathrm{s}}{ }^{(\mathrm{ml}, 2)}\right\}$-two component's Selectivity factor in $\left(\mathbf{I} . \mathrm{K}_{1,2}\right)$.

In this manuscript there is realized the author's theoretical computerized investigation of the $\operatorname{Multi}(\mathbf{n}=\mathbf{6})$-component Mass Transfer (MMT) kinetic process on the basis of the noval advanced NC Model developed with the expanded $\mathbf{k}^{(2)}(\mathbf{6})$-variance. The visual conceptual schemes of the advanced bi-functional $\mathbf{k}^{(2)}-\mathbf{N C}$ Model mentioned are depicted in Figure 1 (S.5). In the manuscript there is considered theoretically the MMT kinetic sorption process inside the bi-functional NC planar $\mathbf{L}$-matrices via the computerized numerical simulation on the basis of the advanced bi-functional $\mathbf{k}^{(2)}-\left\{2_{\mathrm{p}} \mathrm{x}_{\mathrm{m}}\right\} \mathbf{N C}$ Model developed here.

The diffusing ${ }_{(3,4)} \mathrm{p}^{+}$-sorbate principals and the corresponding fixed ${ }_{\mathrm{m}(1,2)}(\mathrm{R} p)^{+}$-principals represent the participants of the two $\left(\mathbf{I}_{1,2}\right)-\mathrm{MAL}_{\mathrm{S}}$ reactions with the paired $\left(\mathbf{I} . \mathrm{K}_{1,2}\right)-\mathrm{MAL}_{\mathrm{S}}$ relationships including the corresponding determinative two $\left\{\mathrm{K}_{\mathrm{S}}^{\mathrm{m} 1,2}\right\}$-Selectivity parameters (Figures 1, S.5).

Thus the advanced $\mathbf{k}^{(2)}(6)-\mathbf{N C}$ Model elaborated comprises the four $\left\{2_{\mathrm{p}} \mathrm{x}_{2}\right\}=\left\{{ }_{(3,4)} \mathrm{p}^{\mathrm{x}} \mathbf{m}_{1,2}\right\}-$ paired principal diffusible ${ }_{(3,4)} \mathrm{p}$ and $\mathbf{m}_{1,2}$-fixed $\left(\mathbf{D}_{1,2}=0\right)$ components participated in the paired selective two $\mathrm{MAL}_{\mathrm{S}}-$ reactions along the paired $\left(\mathbf{I}_{1,2}\right)-\mathrm{MMT}$ route for the paired "sorption $(\mathbf{I} \boldsymbol{a})$-desorption $(\mathbf{I} \boldsymbol{d})$ " $\quad\left\{{ }_{(3,4)} \mathrm{p} \rightleftharpoons \mathbf{m}_{1,2}\right\}$-kinetic mass transformation process together with the corresponding selective paired $\left(\mathbf{I}_{\mathrm{K}} \mathrm{K}_{1,2}\right)-\mathrm{MAL}_{\mathrm{S}}$ relationships as depicted in Figure 1 in S.5.

Figure 1 includes the "sink"(left)-"source" $(r i g h t) \mathbf{J}_{\mathbf{m} 1,2} ; \mathbf{J}_{\mathbf{k},(3.4) \mathbf{p}}$-mass fluxes mechanism illustration (upstairs at Figure 1) of the sorption(left, $\mathbf{I} a)$-desorption(right, $\quad \mathbf{I} d)$ stages during the "association(left)dissociation(right)" $\left(\mathbf{I}_{1,2}\right)$-sorption process (where I $\mathbf{a}$,left-"sink";
Id,right-"source", see Figure 1 near the vertical arrows upstairs). The directions of the vertical arrows (up or down) show the corresponding $\mathbf{J}_{\mathbf{k}, \mathbf{m}, \mathbf{p}}$-mass fluxes (left-"sink"; right-"source" in Figure 1).

The MMT kinetic process is realized in Time (Figure 2: from $(a$, $\left.\mathrm{T}^{1}\right) \square$ to $\left(b, \mathrm{~T}^{3}\right)$ ) across the planar $\mathrm{L}-$ membrane (where $0 \leq \mathrm{L} \leq 1$; to left $(\mathrm{L}=0)$ from right, $(\mathrm{L}=1))$; see Figures 2$)$ as it is demonstrated for the visual example in the two Figure 2 (from $(a, u p)$ to $(\mathrm{b}$, down), where $\mathrm{T}^{1}(a, u p)$ ? $\mathrm{T}^{3}(b$, down $)$.

( $a$, up, $\mathbf{T}^{\prime}$ )

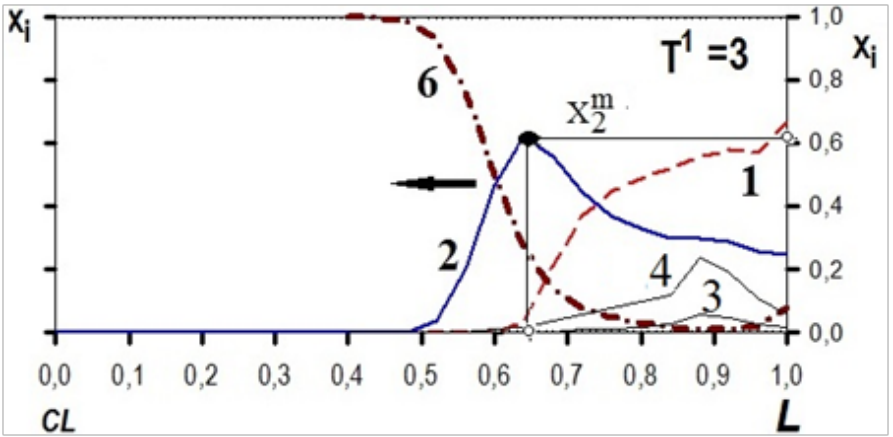

$\left(b\right.$, down, $\left.\mathbf{T}^{3}\right)$

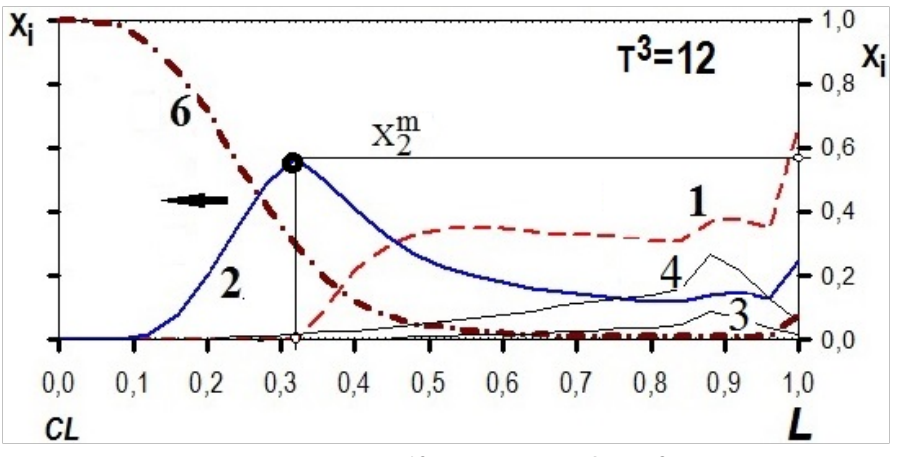

Figure 2 DD-effect, wave $X_{n}\left(L, T^{1,3}\right)$-profiles $\left(a, T^{1} ; b, T^{3}\right)$. "Picture-frames" of $\mathrm{X}_{1-6}\left(L, \mathbf{T}^{1,3}\right)$-travelling waves. Diffusivities $\left\{\mathbf{D}_{3}=0.11 ; \mathbf{D}_{4}=0.055 ; \mathbf{D}_{5}=0.02\right\}$. Selectivities: $K_{s}{ }^{m 1}=400>K_{s}{ }^{m 2}=50$ (dimensionless).

The NC planar L-matrix is presented below by the example of the $\mathrm{NC}$ as the combined Ion Exchanger matrix containing inside the active "NP0 ${ }^{0}$ nanosites" formed as the agglomerates of the NanoParticles $\left(\mathrm{NP}^{0}\right)$. The " $\mathrm{NP}$ - -agglomerates" of the zero charged " $\mathrm{NP}^{0}-\mathrm{Me}^{0 "}$ are included into the NC planar L-matrix, and specified here as the 6th, key ${ }_{\mathbf{k}(6)} \mathrm{R}^{0}$-"NP $\mathrm{N}^{0}$-nanosites". For the clearness the active ${ }_{\mathbf{k}(\boldsymbol{6})} \mathrm{R}^{0}$ nanosites-agglomerates" are shaded in the visual conceptual scheme in Figure 1 (S.5, below).

A number of the visual experimental SEM examples of the bifunctional NC ("Me ${ }^{0}-$ Ion Exchangers") combined NC matrices synthesized in the experiments are demonstrated in the Russian monograph. ${ }^{4}$ The active "NP ${ }^{0}$-nanosites" are fixed inside the $\mathrm{NC} \mathrm{L}-$ matrix during the process of the preliminary experimental NC synthesis (the details of the NC synthesis are presented in the contemporary Russian monograph mentioned. ${ }^{4}$ The MMT NC "sink"-"source" mass transformation mechanism with the corresponding internal $\mathbf{J}_{\mathbf{k} . \mathbf{m} . p}$-fluxes (visually depicted in Figure 1, S.5) are introduced into the mathematical computerized solution of the mass balance differential $\boldsymbol{n}$-Eqns. mentioned. 
The numerical computerized solution of the partial differential $\boldsymbol{n}$-Eqns. system gives in result the multi-component dependences, namely the wave profiles of the various travelling multi-component $\left\{\mathbf{X}_{n}(L\right.$-distance; $\mathbf{T}$-time $\left.)\right\}$-concentration waves. These concentration dependences describe the propagating mode of the interactive $\left\{\mathbf{X}_{n}(L, \mathbf{T})\right\}$-travelling concentration waves of the $\boldsymbol{n}$-componentsparticipants along the $\mathrm{L}$-distance $(0 \leq \mathrm{L} \leq 1)$ in the course of the dimensionless $\mathrm{T}$-time $\left(\mathrm{T}^{i}, i=1,2,3, ..\right)$ during the MMT bi-functional NC kinetic process showing by the visual "pictures-frames" (the examples are depicted in the visual Figure $2\left(\right.$ a up, $\mathbf{T}^{\mathbf{1}} ; \boldsymbol{b}$, down, $\left.\mathbf{T}^{3}\right)$ by the use of a computer (detailes in S.5).

The visual calculated examples of the propagating mode (from $(a, u p)$ to $(b$, down $)$,in Figure 2) of the multi-component $\left\{\mathbf{X}_{n}(L ; \mathbf{T})\right\}-$ traveling concentration waves are represented as the "picture-frames" shown in Figure 2: $\left(a, \mathbf{T}^{\mathbf{1}}\right) ;\left(b, \mathbf{T}^{3}\right)$ in S.6.

The propagating mode of the multi-component $\left\{\mathbf{X}_{n}(L, \mathbf{T}\}-\right.$ concentration waves behaviour together with their interferenceinteraction during the computerized simulation in the combined sorption NC media-matrix gives the fundamental understanding of the MMT NC theoretical problems. Previously the wave approach (here $\mathbf{W}$-concept) has been used rather successfully by the simple previous MMT $\mathbf{k}^{(1)}(\mathbf{5})-\left\{1_{\mathrm{p}}{ }^{\mathrm{x}} 1_{\mathrm{m}}\right\}$ bi-functional NC Model for the MMT NC kinetics with the three $\left\{{ }_{\mathrm{m} 1}(\mathrm{R} p)^{+},{ }_{(3,4)} \mathrm{p}^{+}{ }_{5} \mathrm{R}^{0}\right\}$-principal components in the NC Model for the $\mathbf{k}^{(1)}(\mathbf{5})$-variance in the publications. ${ }^{2,3}$

\section{Fundamental wave (W)-concept for the various multicomponent kinetic and dynamic MMT systems}

The fundamental (W)-concept of "the multi-component waves"1-3 is widely used in the theoretical description for many scientific fields of the multi-component transport for the various kinetic and dynamic MMT systems. The extensive review" ${ }^{3}$ presents the "multi-component waves" (W)-concept which has wide area for the applications in such various research fields as the percolation processes ${ }^{5}$, mechanics of liquids, gas dynamics, theory of burning and even the street traffic. ${ }^{3}$ The $\mathrm{W}$-concept embrace the whole series of the migration phenomena such as chromatography, sedimentation, distillation, electrophoresis and some others. $5,6,7$

The majority of variants of the research fields are reviewed in short in the well known and excellent Helfferich, and Klein theoretical monograph. ${ }^{7}$ That monograph is devoted basically to the ideal sharp or diffusion nonlinear concentration waves interference for the theory of the nonlinear multi-component sorption dynamic systems for the variant of the constant separation coefficients of the componentsparticipants in the theory of multi-component chromatography. ${ }^{7}$ The constancy of the separation coefficients means the formal equivalence of the theory of chromatography in publication ${ }^{7}$ to the variant of the multi-component Langmuir sorption isotherms (or for IEx of equally charged counterions) for the multicomponent sorption equilibria. ${ }^{7}$ The extended review ${ }^{6}$ pays attention to the various nonlinear aspects of the multicomponent sorption dynamics and chromatography.

In the cited publications ${ }^{1-3,8}$ the fundamental "wave Wconcept" is used in the visual demonstrations via the corresponding visualization ("SCA.avi") method (S.6) of the propagating mode of the multi-component $\left\{\mathbf{X}_{n}(L, \mathbf{T})\right\}$-concentration waves during the MMT NC kinetic process. ${ }^{2,3,8}$ The propagation of the multi(n)component $\left\{\mathbf{X}_{n}(\boldsymbol{L}, \mathbf{T})\right\}$-concentration waves is calculated previously by the computer numerical solution of the kinetic mass transfer partial differential $\boldsymbol{n}(\mathbf{5})$-Eqns. for the simple MMT $\mathbf{k}^{(1)}(\mathbf{5})-\left\{1_{\mathrm{p}}{ }^{\mathrm{x}}{ }_{\mathbf{m}}\right\}$ bi-functional NC system mentioned with three $\left\{{ }_{\mathrm{m} 1}(\mathrm{R} p)^{+},{ }_{3} \mathrm{p}^{+},{ }_{5} \mathrm{R}^{0}\right\}-$ principals including the crucial ${ }_{5} \mathbf{R}^{0}$-principal component imtroduced into the consideration in the publications. , $3,8^{2}$

In all researches mentioned the physical sense of the $\left\{\mathbf{X}_{n}(\mathrm{~L}, \mathbf{T})\right\}-$ traveling concentration waves considered may be obviously interpreted and explained as "the propagation of the disturbances along the spatial $\boldsymbol{L}$-coordinate in the course of $\mathbf{T}$-time" following to the known waves definition. ${ }^{9}$

The fundamental wave (W)-concept of the multi-component $\left\{\mathrm{X}_{\mathrm{n}}(\mathrm{L}\right.$-distance;T-time $\left.)\right\}-$ "concentration waves" of the irreversible thermodynamics approach mentioned is widely used in the theoretical description for many scientific fields of the multi-component transport for the various kinetic and dynamic MMT systems. In the publications $s^{2,3,8}$ the fundamental "wave concept" described above (specified here as $\mathbf{W}$ ) is used in the visual demonstrations by the corresponding visualization method presented here with using "SCA. avi"-video-animation-files (see S.6,7 also).

The propagation of the multi $(\boldsymbol{n})-$ component $\left\{\mathbf{X}_{\mathrm{n}}(L, \mathbf{T})\right\}-$ concentration waves is calculated previously by the computerized numerical solution of the mass transfer partial differential $\mathbf{n ( 5 )}$ Eqns. for the MMT combined NC sorption system with the simple bi-functional NC Model which is characterized by the corresponding variance- ${ }^{\text {prev }} \mathbf{k}^{(1)}(\mathbf{5}) .^{2,3,8}$

\section{Noval advanced bi-functional $k^{(2)}(6)-\left\{2_{p} \times 2_{m}\right\}$ NC model developed}

The zero charged author's crucial ${ }_{6} \mathbf{R}^{0}$-principal component (where ${ }_{6} \mathbf{R}^{0}$ is the active "NP0 - nanosites" mentioned,- (shaded in Figure 1, below) is introduced purposely into the noval advanced bi-functional $\mathbf{k}^{(2)}(\mathbf{6})-\left\{2{ }_{\mathrm{p}} \mathbf{2}_{\mathrm{m}}\right\}$ NC Model developed for the further theoretical computerized investigation of the extended $\mathbf{n}(\mathbf{6})$-multi-component mass tranfer NC kinetic process. The fixed crucial and key ${ }_{6} \mathbf{R}^{0}$ principal component plays as before (for $\mathbf{n}=\mathbf{5}$ ) 2,3,8 the cardinal role of the ${ }_{6} \mathbf{R}^{0}$-active "NP0-nano-sites" participated in the formation of the selective "sorption $(\mathbf{I} \boldsymbol{a}-)$-desorption $(-\mathbf{I} \boldsymbol{d})$ " stages of the paired $\left(\mathrm{I}_{\mathrm{K}} \mathrm{K}_{1,2}\right)-\mathrm{MAL}_{\mathrm{s}}$ relationships of the paired $\left(\mathbf{I}_{1,2}\right)-\mathrm{MAL}_{\mathrm{s}}$ reactions for the diffusing ${ }_{(3,4)} \mathrm{p}^{+}$-principals sorbate participants (see relations in Figure 1). Thus the noval bi-functional $\mathbf{k}^{(2)}-\left\{2_{\mathrm{p}}{ }^{\mathrm{2}} \mathbf{2}_{\mathrm{m}}\right\}$ NC Model developed here is described by the paired $\operatorname{MAL}_{\mathrm{S}}\left(\mathbf{I}_{1,2}\right)$ and $\left(\mathbf{I} \cdot \mathrm{K}_{1,2}\right)$ relationships including the two components' $\left\{\mathrm{K}_{\mathrm{S}}{ }^{\mathrm{m} 12}\right\}$-Selectivity factor in $\left(\mathbf{I}_{1,2}\right)$-relationships (in Figure 1, S.5 below).

The fixed ${ }_{\mathbf{m}(1,2)}(\mathbf{R} p)^{+}$-indiffusible $\left(\mathbf{D}_{1,2}=0\right)$ principal complexes, ${ }_{(3,4)} \mathrm{p}^{+}$-sorbate diffusants $\left(\mathrm{D}_{3,4}>0\right)$, and the crucial and key ${ }_{6} \mathbf{R}^{0}$ - fixed principal component $\left(\mathbf{D}_{6}=0\right)$ are participated in the selective paired $\mathrm{MAL}_{\mathrm{S}}$ reactions along $\left(\mathbf{I}_{1,2}\right)$-route with the corresponding paired $\mathrm{MAL}_{\mathrm{S}}$ selective $\left(\mathbf{I}_{\mathrm{K}} \mathrm{K}_{1,2}\right)$-relationships $\left(\left\{\mathrm{K}_{\mathrm{S}}{ }^{\mathrm{m} 12}\right\}\right.$-factor in Figure 1 below).

In the result all the $\boldsymbol{n}$-components investigated are participated in the 6-components' bi-functional ( $\left.\mathbf{I}_{1,2}\right)$, (II)-mass transfer co-routes of the MMT bi-functional NC system (see visual conceptual schemes in Figure 1 considered here) as it follows, namely $\boldsymbol{n}=\left\{_{\mathbf{m}(1,2)}(\mathbf{R} p)^{+}\right.$-fixed principals $\left(D_{1,2}=0\right) ;{ }_{(3,4)} \mathrm{p}^{+}$-sorbate principals-diffusants $\left(\mathbf{D}_{3.4}>0\right) ; \mathrm{j}_{5}{ }^{-}$ coions $\left(\mathbf{D}_{5}>0\right)$, and finally the crucial ${ }_{6} \mathbf{R}^{0}-$ fixed principal " $\mathrm{NP}$ - - nanosites" $\left.\left(\mathbf{D}_{6}=0\right)\right\}$.

The fixed 1,2,6- principal components $\left(\mathbf{D}_{1,2} \mathbf{2}_{\mathbf{6}}=0\right)$ together with the another ${ }_{(3,4)} \mathrm{p}^{+}$-sorbate principals-diffusants participation in the paired $\left(\mathbf{I}_{1,2}\right)$-selective reactions Figure 1 produces the corresponding 
selective multi-n(6)-component "mass transformation" with the crucial "sink"-"source" mechanism ${ }^{1-3,8}$ which is included into the advanced bi-functional $\mathbf{k}^{(2)}(\mathbf{6})-\left\{2_{\mathrm{p}} \mathrm{x}_{\mathrm{m}}\right\} \mathbf{N C}$ Model elaborated here. The key "sink"-"source" $\mathbf{J}_{\mathbf{k}, \mathbf{m}, \mathbf{p}}$-fluxes mechanism is explained visually via the inequalities: $\mathbf{J}_{\mathbf{k}}(\mathbf{I}, \mathbf{I}$, left $)<0 \quad \& \quad \mathbf{J}_{\mathbf{k}}(\mathbf{I}$ d,right $)>0$ in Figure 1 (upstairs).

\section{Results and discussion}

Peculiar $X_{1,2,6-}$-concentration waves inside NC matrix, physical meaning

The MMT NC kinetic process comprises the two types of the MMT: selective paired $\left(\mathbf{I}_{1,2}\right)$-route (with the two component's $\left\{\mathrm{K}_{\mathrm{s}}{ }^{\mathrm{m} 1,2}\right\}$-Selectivity parameter) together with (II)-multi-Diffusivity co-route $\left\{\mathbf{D}_{3-5}\right\}$ depicted in Figure 1 (downstairs). In addition to the MMT multi-Diffusion $\left\{\mathrm{D}_{3-5}\right\}$ (II)-co-route the two diffusing ${ }_{(3,4)} \mathrm{p}^{+}-$ sorbate principal components are the participants of the combined, and complicated processes, i.e. MMT selective $\left(\mathbf{I}_{1,2}\right)$-route in combination with $\left\{\mathrm{D}_{3-5}\right\}$ multi-Diffusion (II) co-route.

Keeping in mind the additional complexity influence in connection with the electric field introduced, electro-neutrality and the NernstPlank relations for the $\mathbf{J}_{\mathbf{p}, \mathbf{m}, \mathbf{k}}$-mass fluxes (Figure 1, upstairs) it is possible to characterize the MMT NC process discussed only by the generalized and approximate description prepared visually via the computerized modeling by the $\left\{\mathbf{X}_{\mathrm{n}}(L, \mathrm{~T})\right\}$-travelling waves interpretation as in Figure 2.(S. 7).

The Selectivity $\left\{\mathrm{K}_{\mathrm{s}}{ }^{(\mathrm{m} 12)}\right\}$ parameters and the paired $\left\{{ }_{(3,4)} \mathrm{p} \rightleftharpoons \mathbf{m}_{1,2}\right\}$ mass transformation of the principal participants for the sorption $(\mathbf{I} a \rightarrow)$ desorption (Id - ) stages (Figure 1, middle and upstaires) bring the Displacement Development (DD) for the $\mathbf{X}_{1,2}$-principal concentration waves for the 1-(displacer), and 2-(displaced) ${ }_{\mathrm{m}(1,2)}(\mathbf{R} p)^{+}$-sorbed principal components (see Figure $2\left(\mathbf{T}^{\mathbf{1}}\right), b\left(\mathbf{T}^{3}\right), \mathbf{S} 6$, below).

The influence of the two principal ${ }_{(3,4)} \mathrm{p}$-sorbate participantsdiffusants brings the ${ }_{(3,4)} \mathrm{p}$-mobility $\left\{\mathrm{D}_{3,4}\right\}$-properties transfer to the peculiar $\mathrm{X}_{\mathrm{m}(1,2)}(L, \mathrm{~T})$-concentration waves for the indiffusible ${ }_{\mathbf{m}(1,2)}$ $(\mathrm{R} p)^{+}$-principal components-complexes $\left(\mathbf{D}_{1,2}=0\right)$. Therefore two Figures 2 (below) demonstrate visually the travelling behavior (from $\mathrm{T}^{1}\left(\right.$ a), down to $\mathrm{T}^{3}(\boldsymbol{b})$ ) for the peculiar $\mathbf{X}_{1,2,6}$ - principal concentration waves including naturally the diffusing travelling for the principal $\mathbf{X}_{3,4^{*}}$-concentration waves.

The DD-effect (shown by two Figures 2 below) is originated from the interference-interaction (via $\left(\mathbf{I}_{1,2}\right) \&\left(\mathbf{I}, \mathrm{K}_{1,2}\right)-\mathrm{MAL}_{\mathrm{S}}$ relations (in Figure 1,S.5) of the three principal $\left\{\mathbf{X}_{\mathrm{m}(1,2), 6}(L, \mathbf{T})\right\}$-concentration waves of the same peculiar type with zero Diffusivities, $\left(\mathbf{D}_{1,2,6}=0\right)$ together with the accessory of the diffusing $\left\{\mathbf{X}_{(3,4)}(L, \mathbf{T})\right\}$-concentration waves for the ${ }_{(3,4)} \mathrm{p}^{+}$-sorbate principal diffusants $\left(\mathrm{D}_{3,4}>0\right)$.

The corresponding conceptual schemes for the bi-functional $\mathbf{k}^{(2)}(\mathbf{6})-\left\{2_{\mathrm{p}} \mathrm{x}_{\mathrm{m}}\right\}$ recent bi-functional NC Model here (conceptually depicted in Figure 1) display distinctly the combination effect of the bi-functional MMT NC sorption system including the main selective "sink"-"source" $\mathbf{J}_{\mathbf{k}, \mathbf{m}, \mathrm{p}}$-fluxes mechanism (depicted in Figure 1, upstairs) with the two (I.K $\mathrm{K}_{1,2}$ )-paired $\mathrm{MAL}_{\mathrm{S}}$ relationships for the selective $\left\{\mathbf{K}_{\mathrm{s}}{ }^{\mathrm{m} 1,2}\right\}$ paired $\left(\mathbf{I}_{1,2}\right)$-MMT route following by the multiDiffusion (II)-co-route influence $\left\{\mathbf{D}_{3,4,5}>0\right\}$.

\section{Displacement development (DD) and peculiar $\left\{X_{1,2,6}\right\}$ concentration waves behavior}

The selective DD-interaction depends on a number of the governing factors (see S.7, also). Thus the interference of the travelling $\left\{\mathbf{X}_{1,2,6}(\mathrm{~L}, \mathbf{T})\right\} \&\left\{\mathbf{X}_{(3,4)}(\mathrm{L}, \mathbf{T})\right\}$-principal concentration waves for the paired $\left\{\mathbf{2}_{\mathrm{p}} \mathbf{X}_{\mathrm{m}}\right\}$-principal participants in the paired selective $\operatorname{MAL}_{\mathrm{S}}\left(\mathbf{I}_{1.2}\right)$-reactions (Figure 1, middle, S.5) depends on the two components' $\left\{\mathrm{K}_{\mathrm{S}}{ }^{\mathrm{m} 1,2}\right\}$-Selectivity $\mathrm{MAL}_{\mathrm{S}}$ factor in $\left(\mathbf{I}, \mathrm{K}_{1,2}\right)$ including the input $\left\{\mathbf{X}_{1.2}{ }^{0}\right\}$-concentration values, and finally on the multiDiffusion $\left\{\mathbf{D}_{3-5}\right\}$-factor along the MMT NC Diffusion $\left\{\mathrm{D}_{3-5}\right\}$ (II) co-route (see Figure 1 and Figures 2; $\mathbf{T}^{1,3}$-series).

The MMT NC frontal process starts when the six input concentrations for the composition mixture, namely $\left\{_{1,2}\left[\mathbf{R} p^{+}\right]^{0} ;\left[{ }_{3,4} \mathrm{p}^{+}\right]^{0}\right.$; $\left.\left[\mathrm{X}_{i 5}{ }^{-}\right]^{0} ;\left[\mathbf{R}_{6}^{0}\right]^{0}\right\}$ enters the MMT $\mathbf{k}^{(2)}(\mathbf{6})$-selective combined NC system inside the L-membrane with the 6-components' composition at the input $\mathrm{L}=1$ (see Figure 2, at $L=1$ ).

The main role for the intensity of the DD-effect is played by the values of the two components' $\left\{\mathrm{K}_{\mathrm{S}}{ }^{\mathrm{m} 1,2}\right\}$-Selectivity factor for the $\mathbf{X}_{1}$ displacer", together with the $\mathbf{X}_{2}$-component displaced.

The intensity of the DD-effect is characterized by the $\mathbf{X}_{2}{ }_{-}$ maximum value for the $\mathbf{X}_{2}$-concentration wave displaced. The maximum $\mathbf{X}_{2}{ }^{\mathrm{m}}$-value is marked by the black -circled sign on the $\mathrm{X}_{2}{ }^{\mathrm{m}}$-peak (at the $\mathrm{L}_{1,3}$-distances marked on L-abscissa by ( )-white points) in Figure $2\left(\mathbf{T}^{1}\right), \boldsymbol{b}\left(\mathbf{T}^{3}\right)$

The effective separation of the indiffusible fixed ${ }_{m(1,2)} \mathbf{X}$-principal components-complexes is dependent on the governing parameters mentioned including in addition the $\left\{\mathbf{D}_{3,4,5}\right\}$-diffusivity values. The displacement of the 2-component by the 1-displacer is especially effective at the initial stage (till $\mathbf{T}^{1} \leq 3$ ) of the Displacement Development process, see Figure 2 (when $\mathbf{T}^{\mathbf{1}} \leq 3$ ).

\section{Discussion of the applied contemporary visualisation \\ Transformation of the final simulation data to multi- colored "SCA.avi" video animation}

For the best perception the calculated numerical multicomponent results (obtained by the computerized MMT NC simulations) are transformed by the subsequent computerized visualisation of the rather complex MMT kinetic NC process by means of the Sci. Computerized Animations (namely by the "SCA.avi" video-animation-file).

The visualization method realised via the multi-colored "SCA. avi" video-animation-files mentioned has been using during more than decade of years for the multi-component travelling $\left.\mathbf{X}_{n}(L ; \mathbf{T})\right\}-$ concentration waves in publications. . $^{2,3,8,10,11}$

The propagating mode of the $\left\{\mathbf{X}_{1-6}(L, \mathbf{T})\right\}$-travelling concentration waves are strongly expressed by the series of the separate "pictureframe"(see two Figure 2), where the visual examples are displayed as the "multi-colored" figures, namely $\left\{\mathbf{X}_{1-6}(L, \mathbf{T})\right\}$-distribution curves (see the visual examples in Figures $2\left(a, \mathbf{T}^{\mathbf{1}}\right)$ or $\left(\boldsymbol{b}, \mathbf{T}^{3}\right)$. The SCA-animation is realized via the multi-colored "SCA.avi" video-animation-file with the including the separate multi-colored "pictures-frames" (Figures $2 a$ or $2 b$ ) which include the $\left\{\mathbf{X}_{\mathbf{n}}\left(\mathrm{L}, \mathbf{T}^{i}\right)\right\}$ concentration waves-profiles arranged in the "SCA.avi" videoanimation-files in the course of the discrete $\mathbf{T}^{i}$-time moments during the MMT NC kinetic process. Here the each of the "SCA. $a v i$ " video-animation-file is assembled sequentially via the separate "multi-colored pictures-frame" in two Figures $2\left(a, \mathbf{T}^{1}\right)$ or $\left(\boldsymbol{b}, \mathbf{T}^{3}\right)$ calculated during the computerized modeling. The travelling $\left\{\mathbf{X}_{(1, \ldots 6)}\right.$ $\left.\left(\mathrm{L}, \mathrm{T}^{i}\right)\right\}$-concentration waves arranged in the course of the discrete $\mathbf{T}^{i}$ times (shown in Figure 2, $\mathbf{T}^{\mathrm{i}}, \mathbf{i}=\mathbf{1}, \mathbf{2}, \mathbf{3} \ldots$ ). The separate "multi-colored $\left\{\mathrm{X}_{n}\left(L, \mathbf{T}^{i}\right)\right\}-$ "captured $i$-video" are prepared previously at the number 


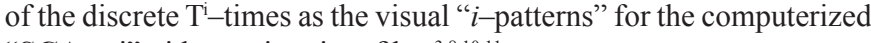
"SCA.avi" video-animation-files. ${ }^{3,8,10,11}$

The execution of the computerized 'SCA.avi' video-animationfile has been used within the many previous and nowadays oral presentations $^{\mathbf{8 , 1 0 , 1 1}}$ for the illustrations of the MMT NC kinetic (or the dynamic) ${ }^{10,11}$ processes (with the examples for a number of the International conferences, for example "IEx 2004-2008-20012", Cambridge, UK) ${ }^{8,10,11}$ For the publication considered here (see as the example publications) $)^{2,3,8,10,11}$ the prepared computerized "SCA.avi" video-animation-files demonstrate (including nowadays author's oral presentations) the continuous Displacement Development (DD-effect) during the animated propagation of the multi-colored" $\left\{\mathbf{X}_{n}(L, \mathbf{T})\right\}$-travelling concentration waves-profiles in the course of the dimensionless T-time. The multi-colored "SCA. avi" videoanimation-file prepared for the NC MMT kinetic ${ }^{3,8} \&$ dynamic $^{10,11}$ processes have been used in the visual perceptible presentations for a number of various sci. audience. , $^{2,8,8,10,11}$

\section{Conclusion}

The application of the fundamental concepts of the irreversible thermodynamics with the inclusion of the recent advanced $\mathbf{k}^{(2)}(\mathbf{6})$ $\left\{2_{\mathrm{p}}{ }^{\mathrm{x}}{ }_{\mathrm{m}}\right\}$ bi-functional NC Model developed gives the noval final theoretical results calculated here by the computerized simulation applied. The presented computer modeling is based on the numerical computerized mathematical solutions of the partial differentials $\boldsymbol{n}(\mathbf{6})$-Eqns describing the multi-component mass balance including the "sink-source" mass $\mathbf{J}_{\mathbf{k}, \mathbf{m}, \mathbf{p}}$-fluxes mechanism (Figures 1,2). The numerical results of the computer simulation are expressed by the $\left\{\mathrm{X}_{n}(L, \mathrm{~T})\right\}$-travelling multi-component concentration wavesdistributions.

The author's experience of the visual oral presentations of the propagating mode of the $\left\{\mathrm{X}_{\mathrm{n}}(L, \mathrm{~T})\right\}$-concentration waves by the multi-colored Sci. Computerized Animations, i.e. by the "SCA. avi" video-animation-files is rather long. ${ }^{2,3,8,10,11}$ The results may be demonstrated alternatively by the "captured video" or "pictureframes" (as in Figures $2\left(a, \mathbf{T}^{\mathbf{1}} ; b, \mathbf{T}^{\mathbf{3}}\right)$ which is more suitable for the printed manuscripts.

The new advanced recent $\mathbf{k}^{(2)}(\mathbf{6})-\left\{2_{\mathrm{p}} \mathrm{x}_{\mathrm{m}}\right\} \mathbf{N C}$ bi-functional Model elaborated here describes the MMT NC kinetic process in the combined NC matrix of the L-membrane. There is considered the original behavior of the peculiar $\left\{\mathbf{X}_{1,2,6}(L, \mathbf{T})\right\}$-travelling concentration waves (where $\mathbf{D}_{1,2,6}=0$ ) in the bi-functional MMT combined NC L-membrane by the computerized simulation discussed (S.6,7). The calculated DD-effect (Figure 2) in the MMT NC kinetics is determined due to the interference-interaction of the peculiar indiffusible $\left\{\mathbf{X}_{1,2,6}(L, \mathbf{T})\right\}$, including the diffusing $\left\{\mathrm{X}_{3,4}(L, \mathbf{T})\right\}$ concentration waves of the 1,2,3,4,6-principal components (where $\mathrm{D}_{1,2,6}=0$ and $\left.\mathrm{D}_{3,4}>0\right)$.

The atypical, and peculiar $\mathrm{X}_{6}$-integral concentration wave behavior for the free $\left.{ }_{6} \mathbf{R}^{0}\right]$ - "NP" ${ }^{0}$-nanosites" (see dashed-dotted $\mathbf{6}$ th-integral curve in the two Figures $2 \mathrm{a}\left(\mathbf{T}^{1}\right), b\left(\mathrm{~T}^{2}\right)$ together with the atypical behavior of the two peculiar travelling $\left\{\mathbf{X}_{1,2}(L, \mathbf{T})\right\}$-concentration waves in the Displacement Development (1st-displacer; 2nddisplaced, Figure 2) have the clear physical meaning: the propagating mode of the peculiar principal $\left\{\mathrm{X}_{1,2,6}(L, \mathbf{T})\right\}$ - concentration waves is determined by the combined behavior of the two MMT co-processes, namely: the selective $\left(\mathbf{I}_{1,2}\right)-\mathrm{MAL}_{\mathrm{S}}$ reactions \& (II)-multi-Diffusion, \{ $\left.\mathbf{D}_{3-5}\right\}$.

The propagating mode of the all peculiar $\left\{\mathrm{X}_{12.6}(L, \mathbf{T})\right\}-$ concentration waves (Figures 2, S.6) during the MMT NC kinetic process is visualized effectively via the created computerized "SCA. avi" video-animation-files. There is the long practice (around 18 years since 2000 including the presentations abroad) $)^{3,8,10,11}$ to use the introduction of the Scientific Animations, video-files "SCA.avi" into the author's oral scientific presentations. The experience of the oral original demonstrations mentioned shows that the computerized simulation results are well perceived by the sci. audience due to the created multi-colored "SCA.avi" video-animation-files as the results of the computerized modeling., $2,3,8,10,11$

\section{Acknowledgments}

None.

\section{Conflict of interest}

Author declares there is no conflict of interest.

\section{References}

1. Kalinitchev A. Kinetic and dynamic chromatographic systems, and models of mass transport: behavior of multicomponent concentration waves. Protection of Metals and Physical Chemistry of Surfaces. 2011;47(6):570-579.

2. Kalinitchev A. New Kinetic computerized Model for Multicomponent Mass Transfer in Bi-functional Matrix of Nanocomposites. Advances in Nanoparticles. 2013;2(2):1-13.

3. Kalinitchev A. Review: MMT kinetics in Nano Composite (NC) bifunctional matrices: $\mathrm{NC}$ selectivity and diffusion concentration waves. NanoTechnol Rev (NTREV). 2014;3(5):467-498.

4. Kravchenko T, Polyansky L. Nano Composites Metal-Ion Exchangers. Nauka; 2009. p. 390. (Monograph inRussian).

5. Rodrigues AE, Tondeur D. Percolation Process: Theory and Application. The Hague: Sijthoff \& Nordhoff; 1981.

6. Kalinitchev A. Nonlinear theory of multi-componentsorption dynamics \& chromatography. J Rus Chem Reviews. 1996;65(2): 95-115.

7. Helfferich F, Klein G. Multicomponent Chromatography. Theory of Interference, New York; 1970. p. 360.

8. Kalinitchev A. Mass Transfer Kinetics Modeling in Bi-functional Ion Exchangers with Chemical Reactions on Active Centers. Cox M, editor. IEX 2012 (El. book) 2012. p. 19-21. Cambridge, UK. London: Soc of Chem Ind (SCI), 2012. p. 1-18. (Thesis; 2012 p. 123-124).

9. Whitham G. Linear and Nonlinear waves. Wiley, NY; 1974. p. 636.

10. Kalinitchev A, Hoell W. Multi-component ion exchange dynamics with the equilibria described by surface complexation theory. Cox M, editor. IEX Technology for Today \& Tomorrow"; 2004. p. 4-8;Cambridge, UK. London: Soc Chem Ind (SCI); 2004. p. 53-58 (Thesis, p.349-356).

11. Kalinitchev A, Hoell W. Theoretical principles of multi-component frontal and displacement elution chromatography on the basis of surface complexation theory. Cox M, editor. Recent Adv in "IEX Theory \& Practice; 2008. p. 9-11; Cambridge, UK. London: Soc Chem Ind (SCI); 2008. p. 85-93. 